\title{
ENDOMORPHISM ALGEBRAS OF ADMISSIBLE $p$-ADIC REPRESENTATIONS OF $p$-ADIC LIE GROUPS
}

\author{
GABRIEL DOSPINESCU AND BENJAMIN SCHRAEN
}

\begin{abstract}
We prove Schur's lemma for absolutely irreducible admissible $p$ adic Banach space (respectively locally analytic) representations of $p$-adic Lie groups. We also prove finiteness results for the endomorphism algebra of an irreducible admissible representation.
\end{abstract}

\section{INTRODUCTION}

In a series of papers [21, 22], 23], 24, Schneider and Teitelbaum defined good abelian categories of representations of $\mathbb{Q}_{p}$-analytic groups on locally convex $p$-adic vector spaces, by imposing suitable "admissibility" conditions. These representations play a crucial role in the, still in its infancy, $p$-adic local Langlands correspondence: deep results of Berger, Breuil, Colmez, Emerton, Kisin and Paškūnas (see [2], [3], 4], [12, [16], to cite only a few) show that the study of irreducible unitary admissible Banach space representations for $\mathrm{GL}_{2}\left(\mathbb{Q}_{\mathrm{p}}\right)$ comes down to the study of irreducible continuous Galois representations $\rho: \operatorname{Gal}\left(\overline{\mathbb{Q}_{p}} / \mathbb{Q}_{p}\right) \rightarrow \mathrm{GL}_{2}\left(\overline{\mathbb{Q}_{p}}\right)$. This can be seen as evidence that these representations are more subtle than their classical analogues (admissible smooth representations or Harish-Chandra modules). For instance, a basic result such as Schur's lemma was unknown in the $p$-adic Banach or locally analytic setting, while the corresponding results for admissible smooth representations or Harish-Chandra modules are rather elementary. In this paper, we prove Schur's lemma in complete generality, for (not necessarily unitary) irreducible admissible Banach space and locally analytic representations of $\mathbb{Q}_{p}$-analytic groups. This was known only for unitary irreducible Banach space representations of $\mathrm{GL}_{2}\left(\mathbb{Q}_{\mathrm{p}}\right)$, having a central character and, for technical reasons, one also needs to assume that $p>3$. This was proved by Paškūnas in [16] in a highly indirect way, by fully using the deformation-theoretic aspects of the $p$-adic local Langlands correspondence and massive computations of extensions between smooth mod $p$ representations.

To be more precise, let $p$ be a prime number, let $L$ be a finite extension of $\mathbb{Q}_{p}$ and let $G$ be a $\mathbb{Q}_{p}$-analytic group of finite dimension (for example, the $\mathbb{Q}_{p}$-points of an algebraic group over $\mathbb{Q}_{p}$ ). A Banach space representation $\Pi$ of $G$ over $L$ is an $L$-Banach space on which $G$ acts by $L$-linear continuous automorphisms. If $H$ is an open compact subgroup of $G$, let $C(H, L)$ be the Banach space of $L$-valued continuous maps on $H$. We say that $\Pi$ is admissible if one can find a closed $H$ equivariant embedding of $\Pi$ into $C(H, L)^{n}$ for some $n \geq 1$ and some $H$. Schneider and Teitelbaum also defined the notion of locally $\mathbb{Q}_{p}$-analytic representations of

Received by the editors July 27, 2011 and, in revised form, December 7, 2012.

2010 Mathematics Subject Classification. Primary 20G05, 20G25, 11E57, 11E95. 
$G$ and that of admissibility for these representations. For the rather technical definitions, see section 2 Our main result is then:

Theorem 1.1. Let $\Pi$ be a topologically irreducible admissible Banach space (resp. locally $\mathbb{Q}_{p}$-analytic) representation of a $\mathbb{Q}_{p}$-analytic group $G$ over $L$. Then

1) The algebra $\operatorname{End}_{L[G]}(\Pi)$ of continuous $G$-equivariant endomorphisms of $\Pi$ is a finite dimensional division algebra over $L$.

2) $\Pi$ is absolutely irreducible (i.e., $\Pi \otimes_{L} L^{\prime}$ is topologically irreducible for any finite extension $L^{\prime}$ of $L$ ) if and only if $\operatorname{End}_{L[G]}(\Pi)=L$.

3) There exists a finite Galois extension $L^{\prime} / L$ and finitely many absolutely irreducible admissible $L^{\prime}$-Banach space (resp. locally $\mathbb{Q}_{p}$-analytic) representations $\Pi_{1}, \Pi_{2}, \ldots, \Pi_{s}$ such that $\Pi \otimes_{L} L^{\prime} \simeq \Pi_{1} \oplus \Pi_{2} \oplus \ldots \oplus \Pi_{s}$.

An obvious consequence of the theorem is that absolutely irreducible admissible Banach space (resp. locally $\mathbb{Q}_{p}$-analytic) representations of $G$ have a central character (resp. a central character and an infinitesimal character). As far as we know, this was unknown even for $\mathrm{GL}_{2}\left(\mathbb{Q}_{\mathrm{p}}\right)$. The hardest step of the proof of Theorem 1.1 is the fact that any $f \in \operatorname{End}_{L[G]}(\Pi)$ is algebraic over $L$. This uses basic structural results of Schneider and Teitelbaum [23] (building on the seminal work of Lazard [14]) and most crucially a deep analogue of Quillen's lemma [18] for almost commutative affinoid $L$-algebras, due to Ardakov and Wadsley [1]. The finiteness (as $L$-vector space) of $\operatorname{End}_{L[G]}(\Pi)$ is a rather tricky combination of Baire's lemma and of a delicate theorem of Kaplansky [11] on PI-division algebras. The second and third part of the theorem are directly inspired from [16, where the case of Banach space representations of $\mathrm{GL}_{2}\left(\mathbb{Q}_{\mathrm{p}}\right)$ is treated. The case of general Banach space representations follows precisely the same lines once the first part of the theorem is proved. The arguments for the case of locally analytic representations are, however, trickier.

We end this introduction by stating a conjecture which lies probably very deep, but which is really one of the results that one would be happy to have in the theory of $p$-adic representations of $p$-adic Lie groups.

Conjecture 1.2. Let $\Pi$ be an absolutely irreducible admissible L-Banach space representation of $G$. Then the space $\Pi_{\text {an }}$ of locally analytic vectors in $\Pi$ is a $G$ representation (topologically) of finite length, having only scalar continuous equivariant endomorphisms (hence having an infinitesimal character).

The subtle point is that $\Pi_{a n}$ is not generally irreducible. This is already seen for parabolic inductions ([25]). Much more delicate examples of this rather surprising situation come from the $p$-adic Langlands correspondence for $\mathrm{GL}_{2}\left(\mathbb{Q}_{\mathrm{p}}\right.$ ) (see [5], 9], [15]). To our knowledge, there is not a single nonabelian $p$-adic Lie group for which the conjecture is known. If $G=\mathrm{GL}_{2}\left(\mathbb{Q}_{\mathrm{p}}\right)$ and if $\Pi$ has a stable lattice whose reduction has finite length (this is automatic if $p>3$ and if $\Pi$ is unitary, by a deep theorem of Paškūnas [16]), one can show the existence of an infinitesimal character for $\Pi_{a n}$ by a very indirect Galois-theoretic argument [6].

1.3. Notations and conventions. We fix a finite extension $L$ of $\mathbb{Q}_{p}$ and we let $O_{L}$ be its ring of integers. The norm $|\cdot|$ on $L$ is normalized such that $|p|=p^{-1}$. For the notions of $\mathbb{Q}_{p}$-analytic group and uniform pro- $p$ group we refer the reader to [7]. For basic facts of nonarchimedean functional analysis, see [20]. If $\Pi$ is a 
continuous representation of $G$ on some locally convex $L$-vector space, $\operatorname{End}_{L[G]}(\Pi)$ will denote the $L$-algebra of continuous $L$-linear $G$-equivariant endomorphisms of $\Pi$.

\section{Preliminaries}

2.1. Duality and admissible Banach representations. Let $G$ be a $\mathbb{Q}_{p}$-analytic group and let $H$ be a compact open subgroup of $G$. A Banach space representation of $G$ over $L$ is an $L$-Banach space $\Pi$ endowed with a continuous action of $G$ (thus the map $G \times \Pi \rightarrow \Pi$ is continuous). Let $O_{L}[[H]]$ be the the completed group algebra of $H$ over $O_{L}$, that is the inverse limit of the group algebras $O_{L}\left[H / H_{1}\right]$, where $H_{1}$ runs over the ordered set of compact open normal subgroups of $H$. By a classical result of Lazard ([14] V.2.2.4), $O_{L}[[H]]$ is a (left and right) noetherian algebra. There is a natural isomorphism of $L$-topological algebras between the algebra of $L$-valued measures $D^{c}(H, L)$ on $H$ (i.e. the topological dual of the space of continuous $L$-valued functions on $H)$ and $L \otimes_{O_{L}} O_{L}[[H]]$. Following Schneider and Teitelbaum [21, an $L$-Banach space representation $\Pi$ of $G$ is called admissible if its topological dual $\Pi^{\prime}$ is a finitely generated module over $L \otimes_{O_{L}} O_{L}[[H]]$. This does not depend on the choice of $H$ and is equivalent to the existence of a closed $H$-equivariant embedding $\Pi \rightarrow C(H, L)^{n}$ for some $n \geq 1$ and some open compact subgroup $H$ of $G$ ([10, Theorem $5.1 .15 \mathrm{i})$ ). We will constantly use the following basic result of Schneider and Teitelbaum ([21], Theorem 3.5).

Theorem 2.2. The duality functor $V \rightarrow V^{\prime}$ induces an anti-equivalence of categories between the category of admissible $L$-Banach space representations of $H$ and the category of finitely generated $L \otimes_{O_{L}} O_{L}[[H]]$-modules.

Theorem 2.2 combined with the fact that $L \otimes_{O_{L}} O_{L}[[H]]$ is noetherian implies that the category of admissible Banach space representations of $G$ is abelian. In particular, continuous equivariant maps between admissible Banach representations of $G$ are strict, with closed image. Hence, if $\Pi_{1}$ and $\Pi_{2}$ are (topologically) irreducible admissible Banach space representations of $G$, then any nonzero $f \in \operatorname{Hom}_{L[G]}\left(\Pi_{1}, \Pi_{2}\right)$ is an isomorphism (the previous discussion shows that $f$ has closed image and the topological irreducibility of $\Pi_{1}$ and $\Pi_{2}$ forces $f$ being injective with dense image. Thus $f$ is bijective and so an isomorphism, by the open image and closed graph theorems).

Remark 2.3. If $G$ is compact, then topological irreducibility for an admissible Banach space representation $\Pi$ of $G$ corresponds to the algebraic simplicity of $\Pi^{\prime}$ as $D^{c}(G, L)$-module (21], Corollary 3.6). On the other hand, if $G$ is no longer compact and $\Pi$ is an irreducible admissible Banach space representation of $G$, then $\Pi^{\prime}$ has no reason to be a simple $D^{c}(H, L)$ module.

2.4. Coadmissible modules and locally analytic representations. We recall in this section a few basic facts about distribution algebras of uniform pro- $p$ groups, following section 4 of 23 . We will freely use the notions of $p$-valuation and ordered basis, for which we refer the reader to loc. cit. Let $G$ be a $\mathbb{Q}_{p}$-analytic group and let $H$ be a uniform open pro- $p$ subgroup of $G$ (such a subgroup always exists by [14]). By section 4.2 of [7], one can find an ordered basis $h_{1}, h_{2}, \ldots, h_{d}$ of $H$ and a $p$-valuation $\omega$ on $H$ such that $\omega\left(h_{i}\right)=1$ if $p>2$ (resp. $\omega\left(h_{i}\right)=2$ if $p=2$ ). We obtain a bijective global chart for the manifold $H$, sending $\left(x_{1}, x_{2}, \ldots, x_{d}\right) \in \mathbb{Z}_{p}^{d}$ to 
$h_{1}^{x_{1}} h_{2}^{x_{2}} \ldots h_{d}^{x_{d}}$ (of course, this is not a group homomorphism). Let $b_{i}=h_{i}-1 \in L[H]$ and, if $\alpha \in \mathbb{N}^{d}$, let $b^{\alpha}=b_{1}^{\alpha_{1}} b_{2}^{\alpha_{2}} \ldots b_{d}^{\alpha_{d}} \in L[H]$. Let $\mathcal{D}(H, L)$ be the algebra of $L$ valued distributions on $H$, as defined in section 2 of [22]. The theory of Mahler expansions shows that the elements of $\mathcal{D}(H, L)$ have unique convergent expansions $\lambda=\sum_{\alpha \in \mathbb{N}^{d}} c_{\alpha} b^{\alpha}$, where $c_{\alpha} \in L$ and $\lim _{|\alpha| \rightarrow \infty}\left|c_{\alpha}\right| \cdot r^{|\alpha|}=0$ for all $r \in(0,1)$ (here $\left.|\alpha|=\alpha_{1}+\ldots+\alpha_{d}\right)$. If $r \in\left(p^{-1}, 1\right) \cap p^{\mathbb{Q}}$, let $\mathcal{D}_{r}(H, L)$ be the completion of $\mathcal{D}(H, L)$ with respect to the norm $\|\lambda\|_{r}=\sup _{\alpha}\left|c_{\alpha}\right| \cdot r^{|\alpha|}$ (thus elements of $\mathcal{D}_{r}(H, L)$ are convergent series $\sum_{\alpha} c_{\alpha} b^{\alpha}$ such that $\left|c_{\alpha}\right| r^{|\alpha|} \rightarrow 0$ as $\left.|\alpha| \rightarrow \infty\right)$. In the case $p=2$, we will prefer the equivalent family of norms defined by ||$\lambda||_{r}=\sup _{\alpha}\left|c_{\alpha}\right| \cdot r^{2|\alpha|}$ (see [19, Prop. 2.1]; the reason for this choice is that we will fully use results of [23], where one uses the norm $\|\lambda\|_{r}=\sup _{\alpha}\left|c_{\alpha}\right| r^{\sum \alpha_{i} \omega\left(h_{i}\right)}$; with our convention and our choice of $\omega$, the norms $\|\cdot\|_{r}$ in this paper agree with the norms $\|\cdot\|_{r}$ in [23]).

One of the main results of [23] (Theorem 4.10; the hypothesis HYP used in loc. cit is precisely the hypothesis that $H$ is a uniform pro- $p$ group, see the remark before Lemma 4.4) is that the natural topological isomorphism of $L$-algebras $\mathcal{D}(H, L) \simeq \lim _{r} \mathcal{D}_{r}(H, L)$ exhibits $\mathcal{D}(H, L)$ as a nuclear Fréchet-Stein algebra. That is, $\mathcal{D}_{r}(H, L)$ are (left and right) noetherian Banach algebras and the transition maps are (right) flat and compact. In section 3 of [23], Schneider and Teitelbaum define the notion of coadmissible module for any nuclear Fréchet-Stein algebra. Let us recall that any such module $M$ is a projective limit of a family $\left(M_{r}\right)$ (with $\left.r \in\left(p^{-1}, 1\right) \cap p^{\mathbb{Q}}\right)$, each $M_{r}$ being a finitely generated $\mathcal{D}_{r}(H, L)$-module such that for $r<r^{\prime}, M_{r}=\mathcal{D}_{r}(H, L) \otimes_{\mathcal{D}_{r^{\prime}}(H, L)} M_{r^{\prime}}$. For any coadmissible module $M$ we have $M_{r}=\mathcal{D}_{r}(H, L) \otimes_{\mathcal{D}(H, L)} M$ and the maps $M \rightarrow M_{r}$ have dense images. Any coadmissible module is naturally a nuclear Fréchet space and all linear maps between coadmissible modules are automatically continuous, with closed image. Finally, the category of coadmissible modules is abelian.

A locally $\mathbb{Q}_{p}$-analytic representation of $G$ is a locally convex $L$-vector space of compact type $V$, endowed with a separately continuous action of $G$ such that orbit maps $g \mapsto g \cdot v$ are in $C^{\text {an }}(G, V)$ (see [22] for the definition of this space of locally $\mathbb{Q}_{p}$-analytic functions on $G$ in this generality). Since the space is of compact type, the action of $G$ is automatically continuous. If $V$ is such a representation and if $H$ is an open compact subgroup of $G$, the action of $G$ extends to a structure of $\mathcal{D}(H, L)$ module on $V^{\prime}$. We say that $V$ is admissible if $V^{\prime}$ is a coadmissible $\mathcal{D}(H, L)$-module for one (equivalently, any) compact open subgroup $H$ of $G$.

Finally, we need to recall a fundamental result of Schneider and Teitelbaum ([23, Theorem 7.1) on the existence of locally $\mathbb{Q}_{p}$-analytic vectors in admissible Banach space representations. This will play a crucial role in the next section. Let $\Pi$ be an admissible $L$-Banach space representation of $G$. A vector $v \in \Pi$ is called locally $\mathbb{Q}_{p^{-}}$analytic if the map $g \rightarrow g \cdot v$ is in $\mathcal{C}^{\text {an }}(G, \Pi)$. The subspace $\Pi_{\text {an }}$ of locally $\mathbb{Q}_{p^{-}}$ analytic vectors of $\Pi$ is naturally a $G$-representation on a space of compact type if we endow it with the topology induced by the embedding $\Pi_{\mathrm{an}} \rightarrow C^{\mathrm{an}}(G, \Pi)$, which sends a vector to its orbit map.

Theorem 2.5. Let $\Pi$ be an admissible $L$-Banach space representation of $G$. Then $\Pi_{\mathrm{an}}$ is a dense subspace of $\Pi$. Moreover, it is an admissible locally $\mathbb{Q}_{p}$-analytic representation of $G$ and for any open compact subgroup $H$ of $G, \Pi_{\text {an }}^{\prime}$ is isomorphic, as a $\mathcal{D}(H, L)$ coadmissible module to $\mathcal{D}(H, L) \otimes_{L \otimes_{O_{L}} O_{L}[[H]]} \Pi^{\prime}$. 


\section{Proof of the main theorem}

3.1. Ardakov-Wadsley's version of Quillen's lemma. The purpose of this section is to prove the following technical result, which will play a crucial role in the proof of Theorem [1.1] The main ingredients are a generalization of Quillen's lemma, due to Ardakov and Wadsley [1] and the computation of the graded ring of $\mathcal{D}_{r}(H, L)$, due to Schneider and Teitelbaum [23]. If $L$ is a finite extension of $\mathbb{Q}_{p}$, we will denote by $O_{L}$ its ring of integers, by $k_{L}$ its residue field and by $\pi_{L}$ a uniformizer of $L$.

Theorem 3.2. Let $H$ be a uniform pro-p group and let $r \in\left(p^{-1}, 1\right) \cap p^{\mathbb{Q}}$. Let $f$ be an endomorphism of a finitely generated $\mathcal{D}_{r}(H, L)$-module $M$ such that each nonzero $g \in L[f]$ is invertible on $M$. Then $f$ is algebraic over $L$.

Proof. Step 1. Choose a finite Galois extension $L^{\prime} / L$ with absolute ramification index $e^{\prime}$ such that $r=p^{-\frac{a}{e^{\prime}}}$ (resp. $r^{2}=p^{-\frac{a}{e^{\prime}}}$ if $p=2$ ) for some integer $a$. Let $A=\mathcal{D}_{r}\left(H, L^{\prime}\right)$, which is a noetherian $L^{\prime}$-Banach algebra for the norm $\|-\| \|_{r}$ (which takes values in $p^{\frac{1}{e^{\prime}} \mathbb{Z}}$, by our choice of $L^{\prime}$ ). Consider the filtration $F \cdot A$ on $A$, induced by the norm (so $F^{s} A$ is the space of those $\lambda \in A$ such that $\|\lambda\|_{r} \leq p^{-s}$ ). As $\|-\|_{r}$ takes values in $p^{\frac{1}{e^{\prime}} \mathbb{Z}}$, we have $\bigcup_{s>0} F^{s} A=\pi_{L^{\prime}} F^{0} A$. Thus, if $\mathrm{gr}^{\cdot}$ denotes the graded ring of $A$ with respect to the filtration $F^{*} A$, then $\operatorname{gr}^{0}(A)$ is naturally isomorphic to $F^{0} A / \pi_{L^{\prime}} F^{0} A$. The proof of Lemma 4.8 of [23] (which heavily depends Theorem 4.5 of loc. cit) shows that we have an isomorphism of rings

$$
\operatorname{gr}^{0}(A) \simeq k_{L^{\prime}}\left[u_{1}, u_{2}, \ldots, u_{d}\right],
$$

where $d=\operatorname{dim} H$. Therefore, $F^{0} A / \pi_{L^{\prime}} F^{0} A$ is naturally a polynomial algebra over $k_{L^{\prime}}$.

Step 2. Using Corollary 8.6 of [1, we obtain the following result: If $N$ is a finitely generated $A$-module and if $f \in \operatorname{End}_{A}(N)$ is an endomorphism such that all nonzero $g \in L^{\prime}[f]$ are invertible on $N$, then $f$ is algebraic over $L^{\prime}$. Indeed, by Step 1 the algebra $A$ satisfies the hypotheses of loc. cit.

Step 3. Finally, let $f$ be as in the statement of the theorem and suppose that $f$ is not algebraic over $L$. Then for each nonzero polynomial $P \in L[X]$, the endomorphism $P(f)$ of $\operatorname{End}_{\mathcal{D}_{r}(H, L)}(M)$ is invertible. If $Q \in L^{\prime}[X]$ is nonzero, then $P=\prod_{\sigma \in \operatorname{Gal}\left(L^{\prime} / L\right)} \sigma(Q)$ is a nonzero polynomial in $L[X]$, hence $P(f)$ is invertible on $M$, and $P(f \otimes \mathrm{Id})$ is invertible on $M \otimes_{L} L^{\prime}$. As $P(f \otimes \mathrm{Id})=\prod_{\sigma \in \operatorname{Gal}\left(L^{\prime} / L\right)} \sigma(Q)(f \otimes \mathrm{Id})$ we conclude that $Q(f \otimes \mathrm{Id})$ is invertible on $M \otimes_{L} L^{\prime}$. By Step 2, $f \otimes$ Id is algebraic over $L^{\prime}$, contradicting the fact that for each nonzero polynomial $Q \in L^{\prime}[X]$, $Q(f \otimes \mathrm{Id})$ is invertible, in particular, nonzero. The result follows.

Remark 3.3. 1) The proof of Corollary 8.6 of [1] is fairly difficult, but the result they prove is much stronger and applies in much more general situations. However, it would be very nice to have a more elementary proof of the corresponding statement in our situation, namely, let $A$ be a Banach $L$-algebra with unit ball $F^{0} A$. Suppose that $F^{0} A / \pi_{L} F^{0} A$ is a polynomial algebra over $k_{L}$. If $M$ is a finitely generated $A$-module and if $f \in \operatorname{End}_{A}(M)$ is such that any nonzero $g \in L[f]$ is invertible on $M$, then $f$ is algebraic over $L$.

2) Completed group algebras do not satisfy the hypotheses of Corollary 8.6 of [1], so when proving Schur's lemma for admissible Banach space representations we will have to use Schneider's and Teitelbaum's results on locally analytic vectors. 
3.4. Algebraicity of $\operatorname{End}_{L[G]}(\Pi)$. The following proposition is one of the key points of the paper. It is essentially a combination of all results recalled in the previous chapter.

Proposition 3.5. Let $\Pi$ be an irreducible admissible L-Banach space (resp. locally $\mathbb{Q}_{p}$-analytic) representation of a $\mathbb{Q}_{p}$-analytic group $G$. Then any $f \in \operatorname{End}_{L[G]}(\Pi)$ is algebraic over $L$.

Proof. Choose an open uniform pro-p-subgroup $H$ of $G$ and fix $r \in\left(p^{-1}, 1\right) \cap p^{\mathbb{Q}}$ such that $\left(\Pi_{\mathrm{an}}^{\prime}\right)_{r} \neq 0$ (resp. $\Pi_{r}^{\prime} \neq 0$ in the locally $\mathbb{Q}_{p}$-analytic case). Such an $r$ exists by Theorem 2.5 in the Banach case and for obvious reasons in the case of locally $\mathbb{Q}_{p}$-analytic representations.

Suppose first that $\Pi$ is a Banach space representation. By Theorem 2.2 any $f \in \operatorname{End}_{L[G]}(\Pi)$ induces an endomorphism $f^{\prime}$ of the finitely generated $D^{c}(H, L)$ module $\Pi^{\prime}$. The natural isomorphism

$$
\left(\Pi_{\mathrm{an}}^{\prime}\right)_{r} \simeq \mathcal{D}_{r}(H, L) \otimes_{D(H, L)} \Pi_{\mathrm{an}}^{\prime} \simeq \mathcal{D}_{r}(H, L) \otimes_{D^{c}(H, L)} \Pi^{\prime},
$$

obtained using Theorem 2.5, allows us to extend $f^{\prime}$ by $\mathcal{D}_{r}(H, L)$-linearity to an element $f_{r}^{\prime}$ of $\operatorname{End}_{\mathcal{D}_{r}(H, L)}\left(\Pi_{\text {an }}^{\prime}\right)_{r}$. This yields an anti-homomorphism $\operatorname{End}_{L[G]}(\Pi) \rightarrow$ $\operatorname{End}_{\mathcal{D}_{r}(H, L)}\left(\Pi_{\text {an }}^{\prime}\right)_{r}$. For locally analytic representations, one still has such an antihomomorphism, essentially by definition of coadmissible modules.

Let $f \in \operatorname{End}_{L[G]}(\Pi) \backslash\{0\}$ and $A=L[f] \subset \operatorname{End}_{L[G]}(\Pi)$. As $\Pi$ is irreducible, the discussion following Theorem 2.2 (resp. analogous properties of the category of admissible locally $\mathbb{Q}_{p}$-analytic representations) shows that any nonzero $g \in A$ is invertible on $\Pi$. As $g \rightarrow g_{r}^{\prime}$ is anti-multiplicative, it follows that $g_{r}^{\prime}$ is an automorphism of the finitely generated $\mathcal{D}(H, L)_{r}$-module $\left(\Pi_{\mathrm{an}}^{\prime}\right)_{r}$ (resp. $\left.\Pi_{r}^{\prime}\right)$. Theorem 3.2 implies that $f_{r}^{\prime}$ is algebraic over $L$, so there exists a nonzero polynomial $P \in L[X]$ such that $P\left(f_{r}^{\prime}\right)=0$. It is easy to see that this forces $P(f)=0$ (otherwise $P(f)$ would be invertible and so $P\left(f_{r}^{\prime}\right)$ would also be invertible). The result follows.

The following proposition is heavily inspired by Lemmas 4.1 and 4.2 of [16]. It is, however, more technically involved in the locally analytic case. Its proof would be much easier (and identical to that of loc. cit) if we knew that an admissible locally analytic representation contains an irreducible closed subrepresentation. For admissible Banach space representations, this is an immediate consequence of the fact that any decreasing sequence of closed subrepresentations is eventually constant (which itself follows easily by duality and the fact that $\mathcal{D}^{c}(H, L)$ is noetherian), but this probably does not happen in the locally analytic case.

Proposition 3.6. Let $\Pi$ be an irreducible admissible Banach space (resp. locally analytic) L-representation of $G$. Then $\Pi$ is absolutely irreducible if and only if $\operatorname{End}_{L[G]}(\Pi)=L$.

Proof. Suppose that $\Pi$ is absolutely irreducible and let $f \in \operatorname{End}_{L[G]}(\Pi)$. Let $P \in$ $L[X]$ be a polynomial killing $f$ (it exists by the previous proposition) and let $L^{\prime}$ be a splitting field of $P$. There exists $c \in L^{\prime}$ such that $f \otimes 1-c$ is not invertible on $\Pi \otimes L^{\prime}$. As $\Pi \otimes L^{\prime}$ is irreducible, it follows that $f \otimes 1=c$. Using the action of $\operatorname{Gal}\left(\mathrm{L}^{\prime} / \mathrm{L}\right)$, it is easy to see that $c \in L$ and so $f=c$ (see [16], Lemma 4.1 for details).

The hard point is the converse implication, which is an easy consequence of the following 
Lemma 3.7. Let $L^{\prime} / L$ be a finite Galois extension and let $\Pi$ be an irreducible admissible Banach space (resp. locally analytic) L-representation of $G$. Then $\Pi_{L^{\prime}}=$ $\Pi \otimes_{L} L^{\prime}$ is a finite direct sum of irreducible admissible $L^{\prime}$-Banach space (resp. locally analytic) representations of $G$.

This lemma yields the desired result, since if $\operatorname{End}_{L[G]}(\Pi)=L$, then $\operatorname{End}_{L^{\prime}[G]}\left(\Pi_{L^{\prime}}\right)$ $=L^{\prime}$ and so $\Pi_{L^{\prime}}$ has to be irreducible. We will prove the lemma in the locally analytic case, which is more difficult (see Lemma 4.2 of [16] for the Banach case). Fix $r \in\left(p^{-1}, 1\right) \cap p^{\mathbb{Q}}$ such that $\Pi_{r}^{\prime} \neq 0$.

Lemma 3.8. For any nonzero $L^{\prime}[G]$ closed subrepresentation $V$ of $\Pi_{L^{\prime}}$ we have $V_{r}^{\prime} \neq 0$.

Proof. We follow some of the arguments in [16], Lemma 4.2. Let $\Gamma=\operatorname{Gal}\left(\mathrm{L}^{\prime} / \mathrm{L}\right)$ and let $r_{\gamma}$ be the continuous automorphism of $\Pi_{L^{\prime}}$ defined by $r_{\gamma}(v \otimes c)=v \otimes \gamma(c)$. We claim that the natural map $\oplus_{\gamma \in \Gamma} r_{\gamma}(V) \rightarrow \Pi_{L^{\prime}}$ is surjective. Its image $X$ is closed in $\Pi_{L^{\prime}}$ (as the image of a map between admissible representations), stable by $G$ and so $X^{\Gamma} \subset \Pi$ is a closed $G$-stable subspace of $\Pi$. But $X^{\Gamma} \neq 0$, as for any $v \in X-\{0\}$ there exists $c \in L^{\prime}$ such that $\sum_{\gamma \in \Gamma} r_{\gamma}(c v) \neq 0$ (by linear independence of characters). Thus $X^{\Gamma}=\Pi$ and so $X=\Pi_{L^{\prime}}$. Now, by duality $\Pi_{L^{\prime}}^{\prime}$ is a submodule of $\bigoplus_{\gamma \in \Gamma} r_{\gamma}\left(V^{\prime}\right)$ and by flatness of $\mathcal{D}_{r}(H, L)$ over $\mathcal{D}(H, L), \Pi_{r}^{\prime} \otimes L^{\prime}$ is a submodule of $\bigoplus_{\gamma \in \Gamma} r_{\gamma}\left(V_{r}^{\prime}\right) \simeq \bigoplus_{\gamma \in \Gamma}\left(V_{r}^{\prime} \otimes_{L^{\prime}, \gamma} L^{\prime}\right)$. In particular, $V_{r}^{\prime} \neq 0$.

Lemma 3.9. $\Pi_{L^{\prime}}^{\prime}$ contains a maximal strict coadmissible $\mathcal{D}\left(H, L^{\prime}\right)$-submodule stable by $G$.

Proof. In this proof, coadmissible submodule will mean a coadmissible $\mathcal{D}\left(H, L^{\prime}\right)$ submodule of $\Pi_{L^{\prime}}^{\prime}$, stable by $G$. By dualizing the result of the previous lemma, it follows that for any coadmissible strict submodule $M$ of $\Pi_{L^{\prime}}^{\prime}$ we have $M_{r} \neq\left(\Pi_{L^{\prime}}\right)_{r}^{\prime}$. Suppose now that $\left(M_{i}\right)_{i \in I}$ is a chain of strict coadmissible submodules of $\Pi_{L^{\prime}}^{\prime}$ and let $N$ be the closure in $\Pi_{L^{\prime}}^{\prime}$ of $\cup_{i} M_{i}$. Then $N$ is a coadmissible submodule of $\Pi_{L^{\prime}}^{\prime}$ and we claim that $N \neq \Pi_{L^{\prime}}^{\prime}$. If we manage to prove this, we are done by Zorn's lemma. As $\left(\Pi_{L^{\prime}}\right)_{r}^{\prime}$ is finitely generated over $\mathcal{D}\left(H, L^{\prime}\right)_{r}$, the union of $\left(M_{i}\right)_{r}$ is a strict submodule of $\left(\Pi_{L^{\prime}}\right)_{r}^{\prime}$. On the other hand, $\bigcup_{i}\left(M_{i}\right)_{r}$ is a sub $\mathcal{D}_{r}(H, L)$ module of $\left(\Pi_{L^{\prime}}\right)_{r}^{\prime}$, thus it is closed (we recall that all submodules of a finitely generated module over a noetherian Banach algebra are closed). On the other hand, by definition $\bigcup_{i}\left(M_{i}\right)_{r}$ is dense in $N_{r}$. Consequently $N_{r} \subset \bigcup_{i}\left(M_{i}\right)_{r}$ and so $N_{r} \neq\left(\Pi_{L^{\prime}}\right)_{r}^{\prime}$. Therefore $N \neq \Pi_{L^{\prime}}$, which finishes the proof of the lemma.

Dualizing the previous lemma, we deduce that $\Pi_{L^{\prime}}$ contains an irreducible closed $L^{\prime}[G]$-subrepresentation $V$. The argument in Lemma 3.8 shows that $\Pi_{L^{\prime}}$ is a quotient of $\bigoplus_{\gamma \in \Gamma} r_{\gamma}(V)$. Each of the representations $r_{\gamma}(V)$ is admissible and irreducible. We easily deduce that $\Pi_{L^{\prime}}$ is a finite direct sum of irreducible admissible representations.

Corollary 3.10. 1) Any absolutely irreducible admissible Banach space representation of a $\mathbb{Q}_{p}$-analytic group has a central character.

2) Any absolutely irreducible admissible locally analytic representation of a $\mathbb{Q}_{p^{-}}$ analytic group has an infinitesimal character. 
3.11. Algebraic Fréchet division algebras and finiteness results. The aim of this section is to prove a noncommutative version of a classical result, stating that a complete algebraic field extension of $\mathbb{Q}_{p}$ is finite over $\mathbb{Q}_{p}$. Combined with Theorem 3.5. this will yield the finiteness property of $\operatorname{End}_{L[G]}(\Pi)$ when $\Pi$ is irreducible. Despite the rather innocent looking statement, we could not find a proof avoiding some pretty difficult results from noncommutative algebra.

Theorem 3.12. Let $A$ be a Fréchet algebra over L. If $A$ is a division algebra and if any element of $A$ is algebraic over $L$, then $A$ is finite dimensional over $L$.

Proof. If $P=\sum_{i} a_{i} X^{i}$ is an element of $L[X]$, define its Gauss norm by $|P|=$ $\max _{i}\left|a_{i}\right|$. Let $F_{n}$ be the set of those $a \in A$ that are killed by some polynomial $f \in L[X]$ of degree at most $n$ and such that $1 / n \leq|f| \leq n$. The restriction on the Gauss norm of the polynomials killing the elements of $F_{n}$, the local compactness of $L$ and the completeness of $A$ imply that $F_{n}$ is closed in $A$. By hypothesis, $A$ is the union of the $F_{n}$ 's, so by Baire's lemma there exists $n$ such that $F_{n}$ contains an open ball $B(x, r)$ of $A$. Fix such $n$. If $u, v \in F_{n}$, then $\left(u^{i}\right)_{0 \leq i \leq n}$ is linearly dependent over $L$ and thus so is the family $\left(u^{i} v\right)_{0 \leq i \leq n}$. Therefore, by [17, Cor. 3.19],

$$
\sum_{\sigma \in S_{n+1}} \varepsilon(\sigma)\left(u^{\sigma(0)} v \cdot u^{\sigma(1)} v \cdot \ldots \cdot u^{\sigma(n)} v\right)=0
$$

That is, there exists a nonzero homogeneous polynomial in noncommutative variables $p(u, v)$ such that $p(u, v)=0$ for all $u, v \in F_{n}$. Let $a, b \in A$ be arbitrary elements. Since $B(x, r) \subset F_{n}$, for all $\lambda_{1}, \lambda_{2} \in L$ of sufficiently large valuation we have $x+\lambda_{1} a, x+\lambda_{2} b \in F_{n}$ and so $p\left(x+\lambda_{1} a, x+\lambda_{2} b\right)=0$. But this remains true for any $\lambda_{1}, \lambda_{2}$, as it is a polynomial identity. We deduce (using homogeneity of $p$ ) that $p(a, b)=0$ for any $a, b \in A$. Therefore, $A$ is a polynomial-identity algebra. By a deep theorem of Kaplansky ([11]), any polynomial-identity division algebra is finite dimensional over its center $Z$. Fix an algebraic closure $\overline{\mathbb{Q}_{p}}$ of $\mathbb{Q}_{p}$. Since $Z$ is an algebraic field extension of $\mathbb{Q}_{p}$, there exists an embedding $Z \rightarrow \overline{\mathbb{Q}_{p}}$ of $\mathbb{Q}_{p}$-vector spaces. By Krasner's lemma, $\overline{\mathbb{Q}}_{p}$ has countable dimension over $\mathbb{Q}_{p}$, so $Z$ has at most countable dimension over $\mathbb{Q}_{p}$. But any Fréchet space of at most countable dimension over $\mathbb{Q}_{p}$ is finite dimensional, as it easily follows from Baire's lemma. We deduce that $Z$ is finite dimensional over $\mathbb{Q}_{p}$, hence that $A$ is finite dimensional over $\mathbb{Q}_{p}$. The result follows.

We are now able to prove the following finiteness result:

Theorem 3.13. If $\Pi$ is an irreducible admissible L-Banach space (resp. locally $\mathbb{Q}_{p}$-analytic) representation of a $\mathbb{Q}_{p}$-analytic group $G$, then $\operatorname{End}_{L[G]}(\Pi)$ is a finite dimensional L-division algebra.

Proof. The discussion following Theorem 2.2 shows that $\operatorname{End}_{L[G]}(\Pi)$ is a division algebra. In view of Theorems 3.5 and 3.12 it is enough to prove that $\operatorname{End}_{L[G]}(\Pi)$ is naturally a Fréchet algebra. For Banach space representations, this is clear, since in this case $\operatorname{End}_{L[G]}(\Pi)$ is a closed subalgebra of $\operatorname{End}_{L}(\Pi)$, which is naturally a Banach algebra for the usual sup norm. Let us assume that $\Pi$ is an admissible locally analytic representation of $G$. Fix a compact open subgroup $H$ of $G$, so the topological dual $\Pi^{\prime}$ is a coadmissible $\mathcal{D}(H, L)$-module. As $\Pi^{\prime} \simeq \lim _{r} \Pi_{r}^{\prime}$ and $\mathcal{D}(H, L) \simeq \lim _{r} \mathcal{D}_{r}(H, L)$, one has a natural isomorphism of $L$-algebras

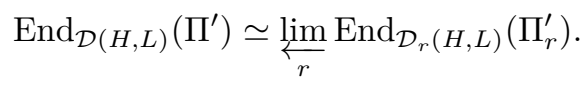


Since $\Pi \rightarrow \Pi^{\prime}$ induces an anti-equivalence between admissible locally analytic representations of $H$ and coadmissible $\mathcal{D}(H, L)$-modules, we have a canonical isomorphism, $\operatorname{End}_{\mathcal{D}(H, L)}\left(\Pi^{\prime}\right) \simeq \operatorname{End}_{L[H]}(\Pi)^{\text {op }}$. Note that $\operatorname{End}_{L}\left(\Pi_{r}^{\prime}\right)$ is naturally a Banach algebra for the sup norm (as $\Pi_{r}^{\prime}$ is a Banach space) and $\operatorname{End}_{\mathcal{D}_{r}(H, L)}\left(\Pi_{r}^{\prime}\right)$ is a closed subspace of it, so again a Banach algebra. Combining these results with the observation that $\operatorname{End}_{L[G]}(\Pi)$ is a closed sub-algebra of $\operatorname{End}_{L[H]}(\Pi)$ yields the desired result. The induced structure of Fréchet algebra on $\operatorname{End}_{L[G]}(\Pi)$ is independent of $H$, as if $H_{1} \subset H_{2}$ are two compact open subgroups of $G$, then $\mathcal{D}\left(H_{1}, L\right)$ (respectively, $\left.\mathcal{D}_{r}\left(H_{1}, L\right)\right)$ is a finite free module over $\mathcal{D}\left(H_{2}, L\right)$ (respectively, $\mathcal{D}_{r}\left(H_{2}, L\right)$ ).

We also note a rather useful consequence of the previous theorem. This has already been observed in [16], Lemma 4.20. There are some extra-assumptions in that reference, precisely because of the lack of the previous result. However, the reader can check that the proof in loc. cit. only uses the fact that $\operatorname{End}_{L[G]}(\Pi)$ is finite dimensional over $L$ and that it also adapts to the case of locally analytic admissible representations.

Corollary 3.14. Let $G$ be a $\mathbb{Q}_{p}$-analytic group and let $\Pi$ be an irreducible admissible Banach space (resp. locally $\mathbb{Q}_{p}$-analytic) representation of $G$. There exist a finite Galois extension $L^{\prime} / L$ and finitely many absolutely irreducible admissible Banach space (resp. locally analytic) representations $\Pi_{1}, \Pi_{2} \ldots, \Pi_{s}$ over $L^{\prime}$ such that

$$
\Pi \otimes_{L} L^{\prime} \simeq \Pi_{1} \oplus \Pi_{2} \oplus \ldots \oplus \Pi_{s} .
$$

\section{ACKNOWLEDGEMENTS}

The first author would like to thank K. Ardakov for kindly and quickly answering some questions concerning [1. He would also like to thank Gaëtan Chenevier and Pierre Colmez for useful discussions about the results of this article.

\section{REFERENCES}

[1] K. Ardakov, S.J. Wadsley, On irreducible representations of compact p-adic analytic groups, to appear in Annals of Mathematics.

[2] Laurent Berger and Christophe Breuil, Sur quelques représentations potentiellement cristallines de $\mathrm{GL}_{2}\left(\mathbf{Q}_{p}\right)$, Astérisque 330 (2010), 155-211 (French, with English and French summaries). MR2642406 (2012i:11053)

[3] Pierre Colmez, La série principale unitaire de $\mathrm{GL}_{2}\left(\mathbf{Q}_{p}\right)$, Astérisque 330 (2010), 213-262 (French, with English and French summaries). MR:2642407 (2011g:22026)

[4] Pierre Colmez, Représentations de $\mathrm{GL}_{2}\left(\mathbf{Q}_{p}\right)$ et $(\phi, \Gamma)$-modules, Astérisque 330 (2010), 281509 (French, with English and French summaries). MR2642409 (2011j:11224)

[5] P. Colmez, La série principale unitaire $p$-adique-vecteurs localement analytiques, to appear in Proceedings of the LMS Durham Symposium 2011.

[6] P. Colmez, G. Dospinescu, Complétés universels de représentations de $\mathrm{GL}_{2}\left(\mathbb{Q}_{p}\right)$, preprint.

[7] J. D. Dixon, M. P. F. du Sautoy, A. Mann, and D. Segal, Analytic pro-p groups, 2nd ed., Cambridge Studies in Advanced Mathematics, vol. 61, Cambridge University Press, Cambridge, 1999. MR $1720368(2000 \mathrm{~m}: 20039)$

[8] Gabriel Dospinescu, Actions infinitésimales dans la correspondance de Langlands locale padique, Math. Ann. 354 (2012), no. 2, 627-657, DOI 10.1007/s00208-011-0736-2. MR2965255

[9] G. Dospinescu, Équations différentielles $p$-adiques et foncteurs de Jacquet localement analytiques, to appear in Proceedings of the LMS Durham Symposium 2011.

[10] M. Emerton, Locally analytic vectors in representations of locally $p$-adic analytic groups, to appear in Memoirs of the AMS.

[11] Irving Kaplansky, Rings with a polynomial identity, Bull. Amer. Math. Soc. 54 (1948), 575580. MR0025451(10,7a) 
[12] Mark Kisin, Deformations of $G_{\mathbb{Q}_{p}}$ and $\mathrm{GL}_{2}\left(\mathbb{Q}_{p}\right)$ representations, Astérisque 330 (2010), 511-528 (English, with English and French summaries). MR2642410(2011e:11185)

[13] Jan Kohlhaase, Invariant distributions on p-adic analytic groups, Duke Math. J. 137 (2007), no. 1, 19-62, DOI 10.1215/S0012-7094-07-13712-8. MR2309143 (2008j:22024)

[14] Michel Lazard, Groupes analytiques p-adiques, Inst. Hautes Etudes Sci. Publ. Math. 26 (1965), 389-603 (French). MR0209286 (35 \#188)

[15] Ruochuan Liu, Bingyong Xie, and Yuancao Zhang, Locally analytic vectors of unitary principal series of $\mathrm{GL}_{2}\left(\mathbb{Q}_{p}\right)$, Ann. Sci. Éc. Norm. Supér. (4) 45 (2012), no. 1, 167-190 (English, with English and French summaries). MR2961790

[16] V. Paškūnas, The image of Colmez's Montréal functor, to appear in Publ. Math. de l'HES, available at http://www.math.uni-bielefeld.de/ ${ }^{\sim}$ paskunas/.

[17] Claudio Procesi, Rings with polynomial identities, Marcel Dekker Inc., New York, 1973. Pure and Applied Mathematics, 17. MR0366968 (51 \#3214)

[18] Daniel Quillen, On the endomorphism ring of a simple module over an enveloping algebra, Proc. Amer. Math. Soc. 21 (1969), 171-172. MR0238892 (39 \#252)

[19] Tobias Schmidt, Auslander regularity of $p$-adic distribution algebras, Represent. Theory 12 (2008), 37-57, DOI 10.1090/S1088-4165-08-00323-3. MR2375595 (2009b:22018)

[20] Peter Schneider, Nonarchimedean functional analysis, Springer Monographs in Mathematics, Springer-Verlag, Berlin, 2002. MR1869547 (2003a:46106)

[21] P. Schneider and J. Teitelbaum, Banach space representations and Iwasawa theory, Israel J. Math. 127 (2002), 359-380, DOI 10.1007/BF02784538. MR.1900706 (2003c:22026)

[22] Peter Schneider and Jeremy Teitelbaum, Locally analytic distributions and p-adic representation theory, with applications to $\mathrm{GL}_{2}$, J. Amer. Math. Soc. 15 (2002), no. 2, 443-468 (electronic), DOI 10.1090/S0894-0347-01-00377-0. MR.1887640(2003b:11132)

[23] Peter Schneider and Jeremy Teitelbaum, Algebras of p-adic distributions and admissible representations, Invent. Math. 153 (2003), no. 1, 145-196, DOI 10.1007/s00222-002-02841. MR 1990669 (2004g:22015)

[24] Peter Schneider and Jeremy Teitelbaum, Locally analytic distributions and p-adic representation theory, with applications to $\mathrm{GL}_{2}$, J. Amer. Math. Soc. 15 (2002), no. 2, 443-468 (electronic), DOI 10.1090/S0894-0347-01-00377-0. MR1887640(2003b:11132)

[25] P. Schneider and J. Teitelbaum, $U(\mathfrak{g})$-finite locally analytic representations, Represent. Theory 5 (2001), 111-128 (electronic), DOI 10.1090/S1088-4165-01-00109-1. With an appendix by Dipendra Prasad. MR 1835001 (2002e:22023)

CMls École Polytechnique, UMR CNRS 7640, F-91128 Palaiseau Cedex, France

Current address: UMPA ENS de Lyon (site Sciences) 46, allée d'Italie, 69364 Lyon cedex 07, France

E-mail address: gabriel.dospinescu@ens-lyon.fr

Laboratoire de Mathématiques de Versailles, UMR CNRS 8100, 45, avenue des États Unis - BÂtiment Fermat, F-78035 Versailles Cedex, France

E-mail address: benjamin.schraen@uvsq.fr 